University of San Diego

Digital USD

Spring 5-26-2018

\title{
Focus on Fitness: Incorporating Exercise into the Treatment of Children and Adolescents with Attention-Deficit Hyperactivity Disorder
}

Kristin Lulich

University of San Diego, kristinpreiser@sandiego.edu

Follow this and additional works at: https://digital.sandiego.edu/dnp

Part of the Child Psychology Commons, and the Nursing Commons

\section{Digital USD Citation}

Lulich, Kristin, "Focus on Fitness: Incorporating Exercise into the Treatment of Children and Adolescents with Attention-Deficit Hyperactivity Disorder" (2018). Doctor of Nursing Practice Final Manuscripts. 66. https://digital.sandiego.edu/dnp/66

This Doctor of Nursing Practice Final Manuscript is brought to you for free and open access by the Theses and Dissertations at Digital USD. It has been accepted for inclusion in Doctor of Nursing Practice Final Manuscripts by an authorized administrator of Digital USD. For more information, please contact digital@sandiego.edu. 
Focus on Fitness: Integrating Exercise into the Treatment of Children and Adolescents with Attention-Deficit Hyperactivity Disorder

Kristin Lulich, MSN, PMHNP-BC, Michael Terry, DNP APRN

University of San Diego 


\begin{abstract}
Background: Psychotropic medications, particularly stimulants are frequently used as the first-line treatment for Attention Deficit Hyperactivity Disorder (ADHD). Many patients and their caregivers have concerns about psychotropic medications or may have only partial response to these agents. Purpose: The evidence based practice (EBP) project introduced the encouragement of aerobic activity into the treatment plan of children and adolescents with ADHD with the aim of reducing symptoms of ADHD by using the NICHQ Vanderbilt Assessment Scale. EBP model: The Iowa Model was used to implement this project. Interventions: Participants were children and adolescents aged 7-17 years receiving psychiatric treatment in an outpatient psychiatric practice with a diagnosis of ADHD. Six children were identified to meet criteria to participate. At the beginning of the project caregivers completed the NICHQ Vanderbilt Assessment Scale. Caregivers were asked about the child's current exercise habits and use of medications. Participants were asked to participate in 30 minutes of aerobic activity at least three days a week for four weeks. Exercise was tracked by caregiver and patient report. At the end of the month caregivers completed a second NICHQ Vanderbilt Assessment Scale and scores were compared to initial scores. Results: Four of the six children were able to fully adhere to the intervention. All participants who adhered to intervention had lower scores at the time of follow up. Participants who adhered to program also reported lower overall scores in comparison to participants who did not adhere to the intervention. Implications for clinical practice: The project illustrates the efficacy of incorporating exercise into the treatment plan of patients with ADHD. This will give providers, patients and/or their caregivers a possible alternative or augmentative
\end{abstract}


treatment modality to traditional medication options. Providers may require training or instruction in how to best implement this with patients and how to allocate time for the intervention during patient visits. Conclusions: Implementing exercise into the treatment planning of children/adolescents with ADHD is a cost effective, well tolerated non-pharmacological intervention that demonstrates improvement in patient outcomes.

Background

The American Academy of Child and Adolescent Psychiatry (2016) recognizes Attention Deficit Hyperactivity Disorder (ADHD) as one of the most common mental health conditions in children. It is a condition that affects over 2.5 million school-aged children in the United States. (Pontifex, Saliba, Picchietti, \& Hillman, 2012). Annual spending on treatment of this condition in the United States is estimated to be between $\$ 143$ billion and $\$ 266$ billion (Abright, 2012). Despite the demand for treatment there is a shortage of child and adolescent psychiatrists, with only 8,300 estimated practicing child and adolescent psychiatrists in the United States (American Academy of Child \& Adolescent Psychiatry, 2016). Symptoms of ADHD as outlined by the Diagnostic and Statistical Manual of Mental Disorders ( $5^{\text {th }}$ ed.; DSM-5; American Psychiatric Association, 2013) include inattentive and/or hyperactive-impulsive symptoms present in multiple settings that clearly interfere with functioning. ADHD is hypothesized to result from a dysregulation of catecholamines. Exercise has been found to increase levels of dopamine, norepinephrine and serotonin, leading to improved attention, focus, distractibility, executive operations, information processing, aggression and hyperactivity (Wigal, Emmerson, Gehricke, \& Galassetti, 2012). The most common treatment for ADHD consists of stimulant medications, such as methylphenidate and 
amphetamine salts. While these medications are highly effective, they often cause significant adverse effects such as anorexia, insomnia, and stunting growth and development. Behavioral modifications, such as exercise are often not included in the treatment plan of children with ADHD, although this has been found as an effective primary or adjunctive treatment with no adverse effects (Heijer et al., 2017) Aerobic activity has been found to improve cognition and behavior in children with ADHD (Gassman, Alves, Santos-Galduriz, \& Galduroz, 2014).

Purpose

The purpose of this EBP was to integrate exercise into the treatment plan of children and adolescents with ADHD with the goal of improving symptoms of ADHD as well as overall health. Exercise has been found to improve response accuracy, stimulus-related processing, enhancements in regulatory processes, as well as improved performance in reading and arithmetic (Pontifex, et al., 2012). Attention, hyperactivity, anxiety, executive functioning, as well as social disorders have also been found to improve with the use of exercise (Cerrillo-Urbina, Hermoso, Sanchez-Lopez, Pardo-Guijarro, Santos-Gomez, \& Martinez-Vizcaino, 2015). Benefits of this intervention can be seen both short and long-term (Ng, Ho, Yong, \& Yeo, 2017). Berwid and Halperin (2012) discuss a major shortfalling of psychotropic medication for ADHD being that while medication is effective at relieving symptoms of the condition, it does not address the underlying neurological mechanisms that are ultimately responsible for symptoms. Whereas exercise has been found to have significant effect on brain function and structure which may lead to longer-term management of symptoms. Integrating exercise into the treatment plan of children and adolescents with ADHD promotes a more holistic approach, as well as offering more options for patients and their families. A 
pilot intervention introducing physical activity was implemented by Smith, Hoza, Linnea, McQuade, Tomb, Vaughn, Shoulberg, \& Hook (2013) showed decreased severity of ADHD symptoms. The literature search for this project included the use of databases Cinahl, The Cochrane Library, PubMed, and GoogleScholar using search terms: ADHD, exercise, Attention Deficit Hyperactivity Disorder, physical activity, aerobic activity, and alternative treatments.

\section{Intervention and Benchmarks}

This evidence based practice (EBP) project identified six individuals aged 7-17, with a diagnosis of predominately inattentive, predominately hyperactive, or combined ADHD seeking treatment in an outpatient private practice psychiatric clinic. Caregivers of the individual were asked to complete the NICHQ Vanderbilt Assessment Scale, which assesses severity of symptoms of inattention, hyperactivity, or both on a likert scale, ranging from $0-4$. There are nine items making criteria for each category for a maximum of 36 points in each area, initial scores were recorded by the nurse practitioner. The caregiver and child were asked about routine exercise habits throughout the week. Education was provided by the nurse practitioner regarding the benefits of exercise in the treatment of ADHD. Participants were asked to engage in a minimum of 30 minutes of aerobic activity at least three times a week for four weeks. At the end of the four weeks, caregivers completed a second NICHQ Vanderbilt Assessment Scale. Scores from pre and post-intervention were compared, and adherence to treatment program was evaluated. The goal of this intervention would be to reduce the total score in each category applicable to the participant by six points. 
Do children and adolescents diagnosed with ADHD, aged 7-10 who have aerobic activity included in their treatment plan, compared to those who do not participate in aerobic activity, have lower scores on the NICHQ Vanderbilt Assessment Scale.

\section{EBP Model}

The Iowa Model of Evidence Based Practice to Improve Quality Care was used in this project.

\section{Results}

Six participants were identified meeting criteria to participate in intervention. Participants were six children and adolescents aged 7-17. Two were seven years old, one was 13, one was 14 , one was sixteen, one was seventeen. There were 4 males and 2 females. Four participants adhered to the intervention. These participants had an average score of 28.7 on the NICHQ Vanderbilt Assessment Scale at the initial evaluation, and an average score of 22.25 on follow up. They participated in at least 30 minutes of aerobic activity an average of 6 times a week. One participant did not adhere to the intervention, the last participant was partially adherent to the intervention. These individuals had an average score of 40 on the initial evaluation, an average score of 34 at the time of follow up and participated in 30 minutes of aerobic activity on average once a week.

\section{Cost-Benefit Analysis}

Costs of implementing this program are minimal. The only costs for this program would include the printing of any educational handouts and reimbursement for provider training. Cost of printing 100 handouts is $\$ 60$, hourly provider reimbursement can be estimated at $\$ 250$ (clinic fee for 60 minute intake for self-pay patients).It can be estimated that annually a patient will need to have a minimum of four scheduled follow ups, with most patients 
presenting for follow up care monthly. The standard self-pay rate for a 30-minute follow up is $\$ 125$. The total cost to train a provider and provide materials to one patient is estimated at $\$ 499.40$. Subtracting the total costs from the estimated revenue of one intake and four follow up visits ( $\$ 750$ - $\$ 250.60$ ) yields a profit of $\$ 499.40$ annually. The program becomes even more cost effective after the initial patient, as there will be no expense of provider training, leaving only the cost of materials, bringing the annual yield for each additional patient to $\$ 749.40$.

\section{Implications for Clinical Practice}

Results from this EBP project demonstrates incorporating exercise into the treatment plan of individuals with ADHD provides an effective alternative or adjunctive treatment for the treatment of this condition. Providers may need to be educated on the benefits of this intervention and how to incorporate it into time already allotted for assessment and evaluation. This EBP project did not exclude participants who had co-occurring disorders such as Intermittent Explosive Disorder, Oppositional Defiant Disorder, Generalized Anxiety Disorder, or Autism Spectrum Disorder, or participants who were not currently taking a stimulant medication. These may be areas be areas to examine further.

\section{Conclusions}

Four out of six participants identified as appropriate participants were able to successfully adhere to the intervention, another participant was partially adherent. Only one participant was completely non-adherent. Individuals who were adherent to the intervention had overall lower scores on the NICHQ Vanderbilt Assessment Scale than individuals who were not adherent. All participants who were adherent to the intervention had improvement in assessment scale scores at the time of follow up. Education and evaluation of the treatment 
program was able to be completed within the established 30-minute appointments. This demonstrates incorporating aerobic activity into the treatment plan of individuals with ADHD is an effective, feasible, well tolerated, non-pharmacological intervention in reducing symptoms of ADHD. 


\section{References}

Abright, A. R. (2012). Estimating the Costs of Attention-Deficit/Hyperactivity Disorder. Journal of the American Academy of Child \& Adolescent Psychiatry 51(10):987-989

American Academy of Child \& Adolescent Psychiatry (2016). Workforce issues. Retrieved from https:/www.aacap.org/aacap/Resources_for_Primary_Care/Workforce_Issues.aspx

American Psychiatric Association. (2013). Diagnostic and statistical manual of mental disorders (5th ed.). Washington, DC: Author.

Berwid, O.G.,\& Halperin, J.M. (2012). Emerging support for a role of exercise in attentiondeficit/hyperactivity disorder intervention planning. Current Psychiatry Reports, 14, 545-551. doi:10.1007/s11920-012-0297-4

Cerrillo-Urbina, A.J., Hermoso, A.G., Sanchez-Lopez, M., Pardo-Guijarro, M.J., Santos-Gomez, J.L., \& Martinez-Vizcaino, V. (2015). The effects of physical exercise in children with attention deficit hyperactivity disorder: a systematic review and meta-analysis of randomized control trials. Child: care, health and development, 41, 779-788. doi:10.111/ cch. 12255

Grassman, V., Alves, M.V., Santos-Galduriz, R.F., \& Galduroz, J.C. (2014). Possible cognitive benefits of acute physical exercise in children with ADHD: A systematic review. Journal of Attention Disorders, 21, 367-371. https://doi.org/10.1177/1087054714526041

Heijer, A.E., Groen, Y., Tucha, L., Fuermaier, A.B., Koerta, J., Lange, K.W., Thome, J., \& Tucha, O. (2017). Sweat it out? The effects of physical exercise on cognition and behavior in children and adults with ADHD: A systematic literature review. Journal of Neural Transmission, 124, 3-26. https://doi.org/10.1007/s00702-016-1593-7

Ng, Q.X., Ho,C.Y., Chan, H.W., Yong, B.,Z., \& Yeo, W. (2017). Managing childhood and adolescent attention-deficit/hyperactivity disorder (ADHD) with exercise: A systematic review. Complementary Therapies in Medicine, 34, 123-128. https://doi.org/10.1016/ j.ctim.2017.08.018

Pontifex, M.B., Saliba, B.J., Raine, L.B., Picchietti, D.L., \& Hillman, C.H. (2013). Exercise improves behavioral, neurocognitive, and scholastic performance in children with attention-deficit/hyperactivity disorder. The Journal of Pediatrics, 162, 543-551. http:// dx.doi.org/10.1016/j.peds.2012.08.036

Smith, A.L., Hoza, B., Linnea, K., McQuade, J.D., Tomb, M., Vaughn, A.J., Shoulberg, E.K., \& Hook, H. (2013). Pilot physical activity intervention reduces severity of ADHD symptoms in young children. Journal of Attention Disorders, 17, 70-82. doi: $10.1177 / 1087054711417395$ 
Wigal, S.B., Emmerson, N., Gehricke, J.G., \& Galassetti, P. (2012). Exercise: Applications to childhood ADHD. Journal of Attention Disorders, 17, 279-290. https://doi.org/ $10.1177 / 1087054712454192$ 